E-mail: jzus@zju.edu.cn

\title{
Correspondence:
}

\section{A discussion on "Tea and human health: biomedical functions of tea active components and current issues"}

\author{
Sheng-rong $\mathrm{SHEN}^{\dagger}$, Sovichea LAY \\ (Department of Food Science \& Nutrition, Zhejiang University, \\ Hangzhou 310058, China) \\ †E-mail: shrshen@zju.edu.cn
}

doi:10.1631/jzus.B1500207

A recent review article on "Tea and Human Health" conducted by Chen and Lin (2015), published in Journal of Zhejiang University-SCIENCE B (Biomedicine \& Biotechnology), was one of the published review papers associated with tea drinking benefits to human health (McKay and Blumberg, 2002; Cabrera et al., 2006; Wolfram, 2007). This paper does not represent a significant advance over previous ones. We think that it is not appropriate that the authors started the article with a very long historical discussion of the evolution of tea rather than discussing the most recent developments; this makes it less interesting. In addition, there are still no conclusive evidence to prove that the bioactive component of tea is really beneficial to human health because the experimental evidence is still conflicting, e.g., Yang et al. (2009) demonstrated that there is no significant difference between the treatment group and control group on the preventive effect of tea. Moreover, there are many reports finding that the relationship between drinking tea habit and cancer is not clear in terms of reduced cancer risks (Bonner et al., 2005; Sun et al., 2006; Li et al., 2008; Zhou et al., 2008). The article also lacked presentation of the modern techniques in clinically experimental medicine against diseases. For example, epigallocatechin gallate (EGCG), the most abundant and active catechin in green tea, was exploited to treat the liver cancer cells of humans and

\footnotetext{
(1) ORCID: Sheng-rong SHEN, http://orcid.org/0000-0003-4096-1693
} (c) Zhejiang University and Springer-Verlag Berlin Heidelberg 2015 animals through cancer nanotechnology, a treatment which is far beyond what the traditional tea-drinking habits would provide of the EGCG bioactive component as normally obtained in the body through oral intake. The results demonstrated that the EGCGfunctionalized nanoparticles exhibited potent anticancer effects on a liver cancer-specific manner (Zhou et al., 2015). The conclusion could be drawn that this technique may serve as the underlying basis of new development strategies on the treatment of liver cancer and other malignancies. Last but not least, the article should have included other biologically active components such as fluoride since the fact is that numerous review papers have already been produced discussing similar compounds, including polyphenols, catechins, flavonoids, and theaflavins. Fluoride is another public health concern that poses a great threat to human health when there is overexposure (WebMD, 2005). It is a natural ability of the tea plant that it can absorb fluoride from surrounding soil. It is estimated that up to $98 \%$ of the accumulated fluoride content in the tea plant is deposited in its leaves, particularly the old ones (Lu et al., 2004). Excessive consumption of fluoride could induce skeletal or dental fluorosis. A newly published article related to skeletal fluorosis due to excessive tea drinking was reported, "A 47-year-old woman had a symptom of bone pain after habitually consumed a pitcher of tea from 100 to 150 tea bags daily for the past 17 years; brewed tea has one of the highest fluoride contents among beverages in the United States. She reported a 5-year history of pain in the lower back, arms, legs, and hips. Because of brittleness, all her teeth were extracted. After scanning radiography, she was eventually diagnosed as skeletal fluorosis," (Kakumanu and Rao, 2013). In conclusion, though bioactive compounds in tea plants are generally considered to have a beneficial effect on human health, 
there is as yet no conclusive scientific evidence on its intrinsic value because of the conflicting results of the research to date. Therefore, more research is needed mainly focusing on not only the traditional way of tea drinking, but also advanced methods, especially the nanotechnological approach. We believe that nanotechnology, using a bioactive tea's component as an agent, could provide stronger supplementary evidence in establishing its real effect on human health.

\section{References}

Bonner, M.R., Rothman, N., Mumford, J.L., et al., 2005. Green tea consumption, genetic susceptibility, PAH-rich smoky coal, and the risk of lung cancer. Mutat. Res., 582(1-2):53-60. [doi:10.1016/j.mrgentox.2004.12.008]

Cabrera, C., Artacho, R., Giménez, R., 2006. Beneficial effects of green tea-a review. J. Am. Coll. Nutr., 25(2):79-99. [doi:10.1080/07315724.2006.10719518]

Chen, Z.M., Lin, Z., 2015. Tea and human health: biomedical functions of tea active components and current issues. $J$. Zhejiang Univ.-Sci. B (Biomed. \& Biotechnol.), 16(2): 87-102. [doi:10.1631/jzus.B1500001]

Kakumanu, N., Rao, S.D., 2013. Skeletal fluorosis due to excessive tea drinking. N. Engl. J. Med., 368(12):1140. [doi:10.1056/NEJMicm1200995]

Li, Q., Kakizuki, M., Kuriyama, S., et al., 2008. Green tea consumption and lung cancer risk: the Ohsaki study. Br. J. Cancer, 99(7):1179-1184. [doi:10.1038/sj.bjc.6604645]

Lu, Y., Guo, W.F., Yang, X.Q., 2004. Fluoride content in tea and its relationship with tea quality. J. Agric. Food Chem., 52(14):4472-4476. [doi:10.1021/jf0308354]

McKay, D.L., Blumberg, J.B., 2002. The role of tea in human health: an update. J. Am. Coll. Nutr., 21(1):1-13. [doi:10.1080/07315724.2002.10719187]

Sun, C.L., Yuan, J.M., Koh, W.P., et al., 2006. Green tea and black tea and colorectal cancer risk: a meta-analysis of epidemiologic studies. Carcinogenesis, 27(7):1301-1309. [doi:10.1093/carcin/bgl024]
WebMD, 2005. Harmful fluoride levels found in instant tea. Available from http://www.webmd.com/food-recipes/2005 0125/harmful-fluoride-levels-found-in-instant-tea [Accessed on Aug. 25, 2015].

Wolfram, S., 2007. Effects of green tea and EGCG on cardiovascular and metabolic health. J. Am. Coll. Nutr., 26(4): 373S-388S. [doi:10.1080/07315724.2007.10719626]

Yang, C.S., Wang, X., Lu, G., et al., 2009. Cancer prevention by tea: animal studies, molecular mechanisms and human relevance. Nat. Rev. Cancer, 9(6):429-439. [doi:10.1038/ nrc2641]

Zhou, Y., Li, N., Zhuang, W., et al., 2008. Green tea and gastric cancer risk: meta-analysis of epidemiologic studies. Asia Pac. J. Clin. Nutr., 17(1):159-165.

Zhou, Y., Yu, Q., Qin, X., et al., 2015. Improving the anticancer efficacy of laminin receptor-specific therapeutic ruthenium nanoparticles (RuBB-loaded EGCG-RuNPs) via ROS-dependent apoptosis in SMMC-7721 cells. ACS Appl. Mater. Interfaces, in press. [doi:10.1021/acsami. 5b02261]

\section{中文概要}

题 目: 关于 “茶与人体健康: 茶叶活性组分的生物医学效 应和当前关注的若干问题” 的讨论

概 要: 茶是世界上最受欢迎的饮料之一。人们享受消费, 获得健康, 普遍认为茶中生物活性物质可以使人 体保持健康, 免遭疾患。但当前的研究结果相互 矛盾, 要断定茶与人类健康的确切关系, 证据不 够充分, 尤其是体外实验与临床结果的不一致, 要明确阐述茶与人体的健康关系为时尚早。因此, 需要更多的研究手段、研究技术和流行病调查, 跨越饮茶的传统方式, 采用更先进的方法, 如纳 米技术等, 以求确丵的科学证据。

关键词: 茶; 健康; 活性组分 